\title{
Observer-based boundary control for 2D Burgers equation
}

\author{
Mehmet Önder Efe
}

TOBB University of Economics and Technology, Department of Electrical and Electronics Engineering, Ankara, Turkey

\begin{abstract}
The 2D Burgers equation has extensively been considered as a benchmark problem by flow control researchers. The flavour of the problem is its multidimensionality and non-linearity, which are the reasons for making the governing equation a good start for developing a well defined understanding of control of flows. This paper demonstrates that an infinite dimensional observer can be designed, and by the aid of the observer, a simple boundary feedback control can be achieved under severe noise and disturbance scenarios. The results are in good compliance with the theoretical claims.
\end{abstract}

Key words: boundary control; Burgers equation; infinite dimensional observer.

\section{Introduction}

Due to its non-linearity and multidimensionality, the 2D Burgers equation constitutes a good benchmark among infinite dimensional systems that can enjoy the standard techniques of classical control theory. Various kinds of Burgers equation have been studied in the past. In Blender (1991), Boules and Eick (2003), Donea and Huerta (2003), Hataue (1998), McDonough and Huang (2004), and Nishinari et al. (2001) simplified version of Navier-Stokes equations given by the partial differential equation (PDE) set $u_{t}+\varepsilon(u \cdot \nabla) u=\mu \nabla^{2} u$ with $u$ being $2 \times 1$ vector function is described as the $2 \mathrm{D}$ Burgers equation. The $2 \mathrm{D}$ Burgers equation is therefore considered a turbulence-free cartoon for Navier-Stokes equations and has been studied in the past

Address for correspondence: Mehmet Önder Efe, TOBB University of Economics and Technology, Department of Electrical and Electronic Engineering, Sögütözü Cad. No. 43 Sögütözü, TR-06560 Ankara, Turkey. E-mail: onderefe@ieee.org 
for modelling traffic flows, shock waves and acoustic transmission. Blender (1991) postulates a method to obtain the solution of the above mentioned PDE set iteratively. Boules and Eick (2003) perform the model reduction with Fourier expansions. In Sirendaoreji (1999), Hietarinta (2000) and Zhu (1996), some other variants of the 2D Burgers equation have been considered with the goal of finding exact solutions under certain circumstances. These types are $\left(u_{t}+u u_{x}-u_{x x}\right)_{x}+u_{y y}=0$ in Sirendaoreji (1999) and Hietarinta (2000), and $u_{t}+u u_{x}+u_{x x}+u_{x x x}=0$ in Zhu (1996). Nishinari et al. (2001) focus on cellular automaton, which is extensively studied for developing models of traffic flow, fluids and immune systems, and therefore a good model to work on is a variant of Burgers equation. In Hataue (1998), the dynamics that arise upon discretization of the 2D Burgers equation is analysed. The effects of a chosen time step $(\Delta t)$ for getting physically reasonable numerical solutions are elaborated. Wescott and Rizwan-uddin (2001) present a computational technique to obtain the numerical solutions of PDEs having non-linear convection terms like the 2D Burgers equation and Navier-Stokes equations. The goal in Wescott and Rizwan-uddin (2001) is to reduce the computation time without giving concessions to accuracy. Boules and Eick (2003) obtain the solution of the Burgers equation for a specific boundary regime and initial conditions. Using a truncated Fourier series expansion yields an autonomous PDE set, the solution of which approximates the numerical solution, and the derived model rebuilds the situation implied by the chosen initial and boundary conditions. When the $1 \mathrm{D}$ version given by $u_{t}=-u u_{x}+u_{x x}$ is taken into consideration, it is seen that a significant amount of research outcome has been reported on modelling and control system design (Burns et al., 2002a,b; Efe and Özbay 2003a, 2004; Efe et al., 2004; Hinze and Volkwein 2002; Krstić, 1999; Liu and Krstić, 2000, 2001; Park and Jang, 2002; Vedantham, 2000). Most of the cited references emphasize the similar difficulties as the motivating factors and focus on the solutions and solvability issues. The current paper, on the other hand, derives an infinite dimensional observer that can be used for boundary control purposes. The contribution of this paper is to demonstrate that simple yet effective observers can be devised to obtain information about the infinite dimensional process, and this information can be used for boundary control purposes. The second section discusses the design of the observer, the third section presents the simulation conditions and obtained results. Concluding remarks are given at the end of the paper.

\section{Design of the observer}

Define $\Omega:=\{(x, y) \mid(x, y) \in[0,1] \times[0,1]\}$ as the physical domain of the process and the observer described by

$$
\begin{gathered}
u_{t}=-k_{1} u u_{x}-k_{2} u u_{y}+\mu u_{x x}+\mu u_{y y} \\
w_{t}=-k_{1} w w_{x}-k_{2} w w_{y}+\mu w_{x x}+\mu w_{y y}+K(u-w)
\end{gathered}
$$


respectively. In (1) and (2), $u=u(x, y, t), w=w(x, y, t)$ and $k_{1}, k_{2}$ and $\mu$ are positivevalued known process parameters, $K$ is the observer gain. The boundary conditions are specified by

$$
\begin{aligned}
& u_{y}(x, 0, t)=\beta_{0} u(x, 0, t), \quad w_{y}(x, 0, t)=\beta_{0} w(x, 0, t) \\
& u_{x}(x, 1, t)=-\beta_{1} u(x, 1, t), \quad w_{y}(x, 1, t)=-\beta_{1} w(x, 1, t) \\
& u_{x}(0, y, t)=\zeta_{0} u(0, y, t), \quad w_{x}(0, y, t)=\zeta_{0} w(0, y, t) \\
& u_{x}(1, y, t)=-\zeta_{1} u(1, y, t), \quad w_{x}(1, y, t)=-\zeta_{1} w(1, y, t)
\end{aligned}
$$

where $\zeta_{0}, \zeta_{1}, \beta_{0}$ and $\beta_{1}$ are positive numbers. The problem is to reconstruct $u(x, y, t)$ over $\Omega$ by designing an observer, whose variable is $w(x, y, t)$, and to perform observerbased feedback control through boundary (corner) excitations.

Theorem 2.1. For the $2 D$ Burgers equation given in (1), an infinite dimensional observer having the structure given by (2) can be designed if and only if there exists $a \lambda>0$ such that $\left|k_{1} \frac{\partial\left(u^{2}-w^{2}\right)}{\partial x}+k_{2} \frac{\partial\left(u^{2}-w^{2}\right)}{\partial y}\right| \leq \lambda|u-w|$ is satisfied. Such an observer with a positive $K \in \mathfrak{R}$ results in exponentially stable reconstruction over $(x, y, t) \in[0,1] \times[0,1] \times[0, \infty)$.

Proof. Consider the Lyapunov function candidate

$$
V=\frac{1}{2} \int_{0}^{1} \int_{0}^{1}(u(x, y, t)-w(x, y, t))^{2} d x d y
$$

For the sake of the simplicity, we drop the arguments of the variables $u$ and $w$, and take the time derivative of the Lyapunov function candidate above. This yields

$$
\begin{aligned}
\dot{V} & =\int_{0}^{1} \int_{0}^{1}(u-w)\left(u_{t}-w_{t}\right) d x d y \\
& =\int_{0}^{1} \int_{0}^{1}(u-w)\left(-k_{1} u u_{x}-k_{2} u u_{y}+\mu u_{x x}+\mu u_{y y}+k_{1} w w_{x}+k_{2} w w_{y}\right. \\
& \left.-\mu w_{x x}-\mu w_{y y}-K(u-w)\right) d x d y \\
& =-2 K V+\int_{0}^{1} \int_{0}^{1}(u-w)\left(-k_{1} u u_{x}-k_{2} u u_{y}+\mu u_{x x}\right. \\
& \left.+\mu u_{y y}+k_{1} w w_{x}+k_{2} w w_{y}-\mu w_{x x}-\mu w_{y y}\right) d x d y
\end{aligned}
$$

Set $s:=u-w$, we have

$$
\begin{gathered}
\dot{V}=-2 K V+\mu \int_{0}^{1} \int_{0}^{1}\left(s s_{x x}+s s_{y y}\right) d x d y-\frac{1}{2} \int_{0}^{1} \int_{0}^{1}(u-w) \\
\left(k_{1} \frac{\partial\left(u^{2}-w^{2}\right)}{\partial x}+k_{2} \frac{\partial\left(u^{2}-w^{2}\right)}{\partial y}\right) d x d y
\end{gathered}
$$


Using the boundary conditions in (3), it is straightforward to show that the following equality holds true:

$$
\begin{aligned}
\int_{0}^{1} \int_{0}^{1} s s_{x x} d x d y & \left.=\int_{0}^{1} s(1, y) s_{x}(1, y)-s(0, y) s_{x}(0, y)\right) d y-\int_{0}^{1} \int_{0}^{1} s_{x}^{2} d x d y \\
& =-\int_{0}^{1} \zeta_{1} s(1, y)^{2}+\zeta_{0} s(0, y)^{2} d y-\int_{0}^{1} \int_{0}^{1} s_{x}^{2} d x d y
\end{aligned}
$$

Likewise we have,

$$
\begin{aligned}
\int_{0}^{1} \int_{0}^{1} s s_{y y} d x d y & \left.=\int_{0}^{1} s(x, 1) s_{y}(x, 1)-s(x, 0) s_{y}(x, 0)\right) d x-\int_{0}^{1} \int_{0}^{1} s_{y}^{2} d x d y \\
& =-\int_{0}^{1} \beta_{1} s(x, 1)^{2}+\beta_{0} s(x, 0)^{2} d x-\int_{0}^{1} \int_{0}^{1} s_{y}^{2} d x d y
\end{aligned}
$$

Utilizing these results with the assumption of the theorem lets us rewrite (6) as follows:

$$
\begin{aligned}
\dot{V} & =-2 K V-\mu \int_{0}^{1} \zeta_{1} s(1, y)^{2}+\zeta_{0} s(0, y)^{2} d y-\mu \int_{0}^{1} \beta_{1} s(x, 1)^{2}+\beta_{0} s(x, 0)^{2} d x \\
& -\mu \int_{0}^{1} \int_{0}^{1}\left(s_{x}^{2}+s_{y}^{2}\right) d x d y-\frac{1}{2} \int_{0}^{1} \int_{0}^{1}(u-w)\left(k_{1} \frac{\partial\left(u^{2}-w^{2}\right)}{\partial x}+k_{2} \frac{\partial\left(u^{2}-w^{2}\right)}{\partial y}\right) d x d y \\
& \leq-2 K V-\mu \int_{0}^{1} \zeta_{1} s(1, y)^{2}+\zeta_{0} s(0, y)^{2} d y-\mu \int_{0}^{1} \beta_{1} s(x, 1)^{2}+\beta_{0} s(x, 0)^{2} d x \\
& -\mu \int_{0}^{1} \int_{0}^{1}\left(s_{x}^{2}+s_{y}^{2}\right) d x d y+\frac{1}{2} \int_{0}^{1} \int_{0}^{1}|u-w|\left|k_{1} \frac{\partial\left(u^{2}-w^{2}\right)}{\partial x}+k_{2} \frac{\partial\left(u^{2}-w^{2}\right)}{\partial y}\right| d x d y \\
& \leq-2 K V-\mu \int_{0}^{1} \zeta_{1} s(1, y)^{2}+\zeta_{0} s(0, y)^{2} d y-\mu \int_{0}^{1} \beta_{1} s(x, 1)^{2}+\beta_{0} s(x, 0)^{2} d x \\
& -\mu \int_{0}^{1} \int_{0}^{1}\left(s_{x}^{2}+s_{y}^{2}\right) d x d y+\frac{1}{2} \int_{0}^{1} \int_{0}^{1} \lambda|u-w|^{2} d x d y \\
& =-(2 K-\lambda) V-\mu \int_{0}^{1} \zeta_{1} s(1, y)^{2}+\zeta_{0} s(0, y)^{2} d y-\mu \int_{0}^{1} \beta_{1} s(x, 1)^{2}+\beta_{0} s(x, 0)^{2} d x \\
& -\mu \int_{0}^{1} \int_{0}^{1}\left(s_{x}^{2}+s_{y}^{2}\right) d x d y \\
& <0
\end{aligned}
$$

This result-shows that the state of the non-linear process in (1) can be reconstructed by the non-linear observer in (2). Since the condition $\left|k_{1} \frac{\partial\left(u^{2}-w^{2}\right)}{\partial x}+k_{2} \frac{\partial\left(u^{2}-w^{2}\right)}{\partial y}\right| \leq \lambda|u-w|$ needs to be satisfied, we can claim that the stability proof is valid only in a local region characterized by the inequality. The next section presents the practical implications of the theorem and its proof above. 


\section{Boundary control of the system}

In this section, we consider the observer-based boundary control of the system in (1). For this purpose, we choose the $(x, y)=(0,0)$ corner as the entry for control excitation denoted by $\gamma_{00}(t)$, and other three corners as the entries for disturbances, $i e, \gamma_{01}(t), \gamma_{10}(t)$ and $\gamma_{11}(t)$. Once the corner conditions are specified, the numerical solution for the boundaries of the domain $\Omega$ are obtained. For example, we solve the equation set

$$
\begin{gathered}
u_{t}=-k_{1} u u_{x}+\mu u_{x x} \\
w_{t}=-k_{1} w w_{x}+\mu w_{x x}+K(u-w)
\end{gathered}
$$

along $x=0$ and $x=1$ segments. A similar strategy is followed for other two segments of the boundary. The numerical solution is obtained by using Crank-Nicholson implicit method (Farlow, 1993). The boundary conditions in (3) are forced and the interior grid solution of the PDE set in (1)-(2) are obtained. The settings of the simulation are tabulated in Table 1, where the process parameters have been chosen in such a way that a simple numerical solver can provide the solution accurately. After a few trials, the time step size and the spatial step size have been refined experimentally.

In order to demonstrate that the observer works well under noisy observations of $u(x, y, t)$, a normally distributed measurement noise corrupts the read quantity, $u$. The controlled variable is $u\left(x_{m}, y_{m}, t\right)$ with $x_{m}$ and $y_{m}$ being the co-ordinates of the arbitrarily chosen measurement location. The control problem is to force $u\left(x_{m}, y_{m}, t\right)$ towards a reference signal $r(t)$ at $(x, y)=\left(x_{m}, y_{m}\right)$. For this purpose, we have set $\left(x_{m}, y_{m}\right)=(0.5172,0.5172)$. As the reference signal, we utilize $r(t)=$ $\operatorname{sign}(\sin (2 \pi 2 t))$. This choice of the reference signal is deliberate as it excites the shock response of both the process and observer, and makes it easy to see the steady-state behaviour.

Table 1 Simulation settings

\begin{tabular}{lc}
\hline$k_{1}$ & 5 \\
$k_{2}$ & 5 \\
$\mu$ & 10 \\
$\zeta_{0}$ & 1 \\
$\zeta_{1}$ & 1 \\
$\beta_{0}$ & 1 \\
$\beta_{1}$ & 1 \\
$\Delta_{t}$ & $1 \mathrm{~ms}$ \\
$T$ & $1 \mathrm{~s}$ \\
$K$ & 10 \\
$N_{x}$ & 30 \\
\hline
\end{tabular}


Aside from the reference signal's step-like nature, the disturbance signals are set as

$$
\begin{aligned}
& \gamma_{01}(t)=0.1 \operatorname{sign}(\sin (2 \pi 20 t)) \\
& \gamma_{10}(t)=0.1 \operatorname{sign}(\sin (2 \pi 17 t)) \\
& \gamma_{11}(t)=0.1 \operatorname{sign}(\sin (2 \pi 15 t))
\end{aligned}
$$

where we inject abruptly changing signals at different frequencies. This kind of a disturbance scenario lets us obtain a rich set of disturbance effect at the fixed location $\left(x_{m}, y_{m}\right)$.

A last issue to figure out is the reconstruction performance of the observer. For this purpose, we define the following integral as the measure of performance,

$$
Q(t)=\int_{0}^{1} \int_{0}^{1}(u(x, y, t)-w(x, y, t))^{2} d x d y
$$

The controller that closes the feedback loop is given by

$$
\gamma_{00}(t)=K_{i} \int_{0}^{t}\left(r(\sigma)-w\left(x_{m}, y_{m}, \sigma\right) d \sigma\right)
$$

where $K_{i}=80$ is the integral controller gain. In Figure 1, a matrix of subplots is depicted with a circle indicating the location $\left(x_{m}, y_{m}\right)$. The plots on the left column are for $u(x, y, t)$ and those on the right column are for $w(x, y, t)$. The first four rows are the snapshots from both processes at $t=1,3,10$ and $20 \mathrm{~ms}$ instants. The last row is for the final time, $T=1$. Clearly, the transient phase passes very quickly and a convergence is observed. This fact is further visible in the bottom right subplot of Figure 2, where the convergence is achieved after the first $50 \mathrm{~ms}$ time.

Looking at the results in Figure 2, a good tracking is seen in the top left subplot, where the reference signal $r(t)$ (thick curve), $u\left(x_{m}, y_{m}, t\right)$ (solid curve) and $w\left(x_{m}, y_{m}, t\right)$ (dashed curve) are plotted together. The process variable and the observer variable are almost indistinguishable, which is a numerical evidence to the analytical claims and existence of a $\lambda>0$. In the top right subplot, this difference is shown, where the convergent behaviour is noteworthy. The bottom left subplot shows the control signal $\left(\gamma_{00}(t)\right)$ that is applied from the corner $(x, y)=(0,0)$. Unsurprisingly, although we impose unit magnitude reference signal at the measurement point, the signal that needs to be applied from the corner is higher in magnitude than the reference. This is because the other three corners are driven by small magnitude disturbances, and the control signal magnitude needs to be larger as the measurement point gets away from the control entry, ie, $(x, y)=(0,0)$. A natural consequence of this is the sufficiency of relatively small control effort in the close neighbourhood of the control entry, and large control effort in the vicinity of locations where the solution is independently specified. For example, in the vicinity of $(x, y)=(1,1)$, the control signal must overcome the effect of corner condition, and naturally the control effort is expectedly large. 

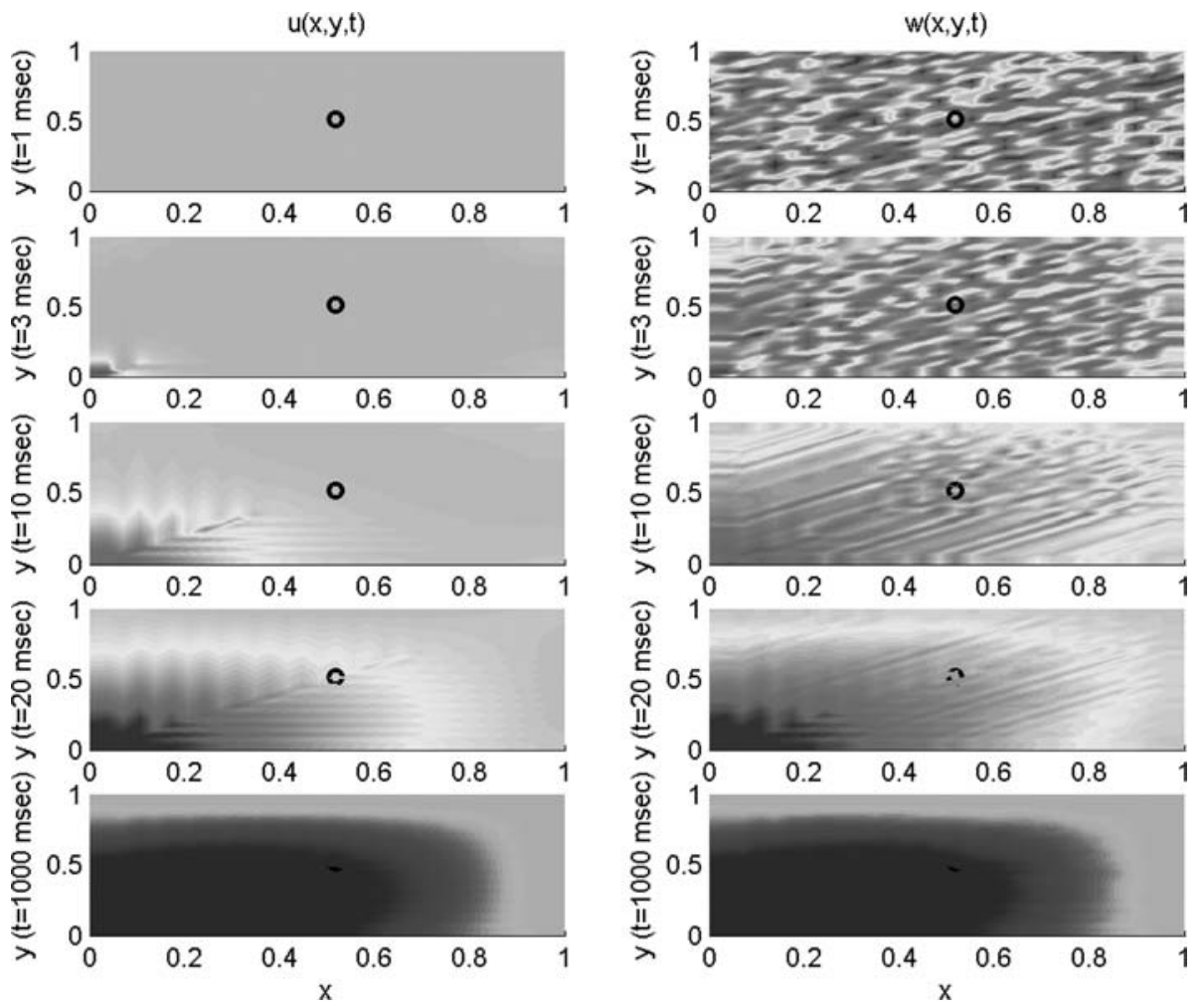

Figure 1 Simulation results with integral controller

Clearly, a last question in this section should ask whether it was difficult to find such a parameter set that works well. The answer is yes, after a few trials, a good resolution parameter $\left(N_{x}\right)$ and a good observer gain $(K)$ can be set together with a controller gain $\left(K_{i}\right)$. We set these values by trial and error. As emphasized by Aström, if the essential dynamics of the system seem to be first-order, a PID-type controller variant should be the first choice (Aström and Hägglund, 1995). According to this, we examined the $\mathrm{P}$, I and D effects through a set of trials. It is observed that the derivative action introduces unnecessarily large control signals due to the noise on $w\left(x_{m}, y_{m}, t\right)$; on the other hand, the proportional action introduces high-frequency components into the control signal that is reflected adversely to the tracking performance. In the end, we confined ourselves to the pure integral action.

\section{Conclusions}

This paper demonstrates that a non-linear infinite dimensional observer can be designed for a non-linear infinite dimensional process. Despite the spatial continuity, under mild assumptions, the design problem enjoys the classical approaches of control theory. A simple yet successful boundary control has been demonstrated. The results indicate that the observer reconstructs the process state in a globally exponentially 

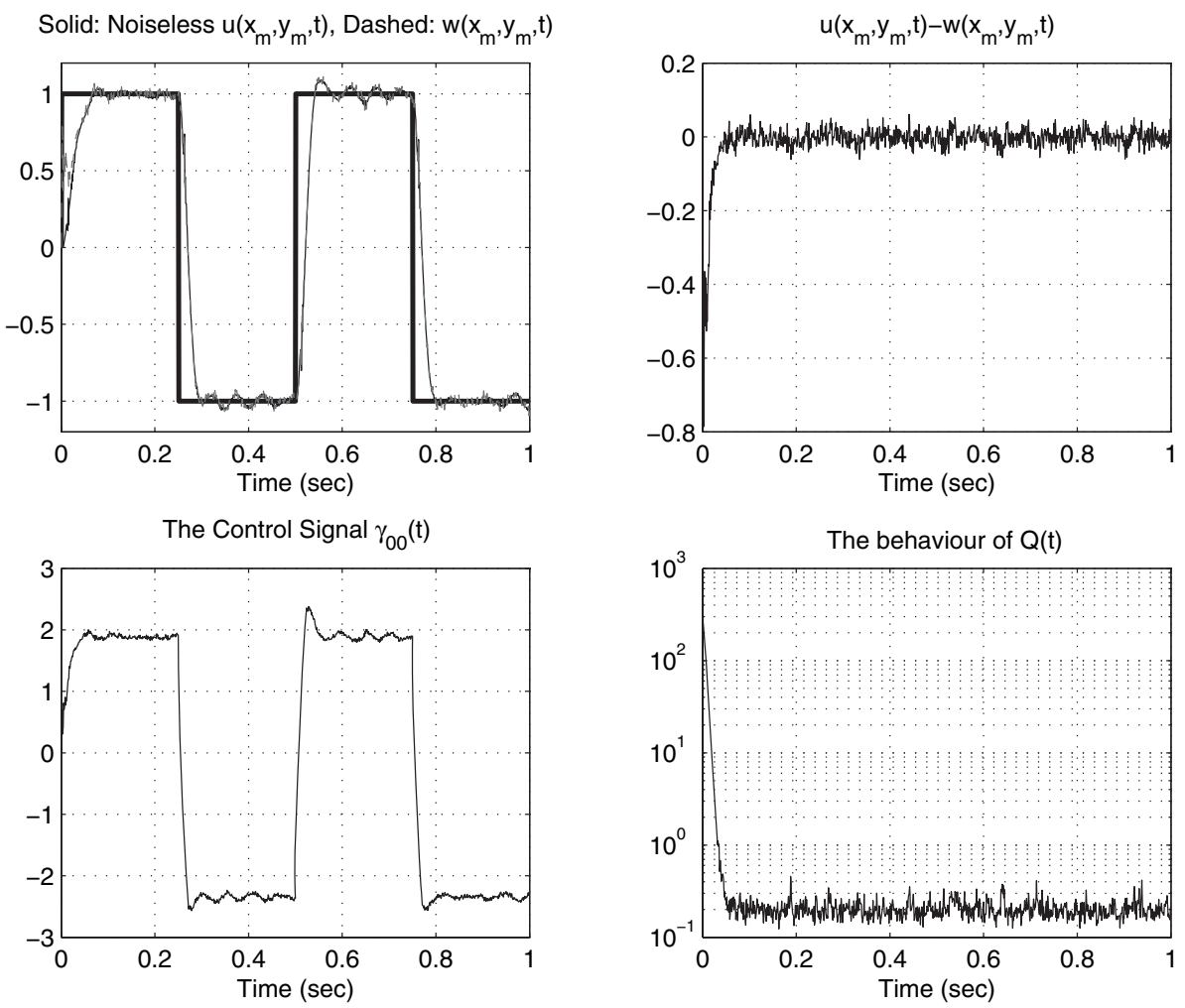

Figure 2 Simulation results with integral controller

stable evolution, and the closed-loop control performance is satisfactory under severely corrupted measurements and corner disturbances. The computational advantages of the controller is another prominent feature that deserves emphasis. The contribution of this paper is the method followed to devise a non-linear observer for a non-linear infinite dimensional problem.

Future research aims to demonstrate a strategy for complicated forms of Burgers equation as in Blender (1991), Boules and Eick (2003), Donea and Huerta (2003), Hataue (1998), and McDonough and Huang (2004), and Nishinari et al. 2001). The contribution at large is to underline the design possibility of observers and observerbased controllers for flow and fluid problems.

\section{Acknowledgements}

This work was supported in part by the AFRL/VA and AFOSR through the Collaborative Center of Control Science at the Ohio State University (Contract F3361501-2-3154) and in part by TOBB ET \“u BAP Program (Contract ET \“u_BAP_2006/04).

The author would like to thank Prof. H. Özbay, Prof. Mo Samimy, Dr J.H. Myatt, Dr J. DeBonis, Dr R.C. Camphouse, Dr P. Yan, X. Yuan and E. Caraballo for fruitful discussions in devising the presented work. 


\section{References}

Aström, K.J. and Hägglund, T.H. 1995: PID controllers: theory, design and tuning. ISA - The Instrumentation, Systems, and Automation Society.

Blender, R. 1991: Iterative solution of nonlinear partial-differential equations. Journal of Physics A: Mathematical and General 24, L509-12.

Boules, A.N. and Eick, I.J. 2003: A spectral approximation of the two-dimensional Burgers equation. Indian Journal of Pure $\mathcal{E}$ Applied Mathematics 34, 299-309.

Burns, J.A., King, B.B. and Zietsman, L. 2002a: On the computation of singular functional gains for linear quadratic optimal boundary control problems. Proceedings of the 3rd Theoretical Fluid Mechanics Meeting, St. Louis, 24-26 June, AIAA, 2002-3074.

Burns, J.A., King, B.B., Rubio, A.D. and Zietsman, L. 2002b: Functional gain computations for feedback control of a thermal fluid. Proceedings of the $3 \mathrm{rd}$ Theoretical Fluid $\mathrm{Me}$ chanics Meeting, St. Louis, 24-26 June, AIAA, 2002-992.

Donea, J. and Huerta, A. 2003: Finite element methods for flow problems John Wiley \& Sons, 252-53.

Efe, M.Ö. and Özbay, H. 2003a: Integral action based Dirichlét boundary control of Burgers equation. Proceedings of the IEEE International Conference on Control Applications (CCA'2003), Istanbul, 23-25 June, 1267-72.

Efe, M.Ö., Yuan, X., Özbay, H. and Samimy, M. 2004: Interpolating local models of POD using fuzzy decision mechanisms. 9th Mechatronics Forum International Conference, 30 August 30-1 September, Ankara, Turkey, 347-56.

Efe, M.Ö. and Özbay, H. 2004: Low dimensional modeling and Dirichlét boundary controller design for Burgers equation. International Journal of Control 7, 895-906.

Farlow, S.J. 1993: Partial differential equations for scientists and engineers. Dover Publications, 317-22.

Hataue, I. 1998: Mathematical and numerical analyses of dynamical structure of numerical solutions of two-dimensional fluid equations. Journal of the Physical Society of Japan 67, 1895-911.
Hietarinta, L. 2000: Comments on 'Exact solutions of the teo-dimensional Burgers equation'. Journal of Physics A: Mathematical and General 33, 5157-58.

Hinze, M. and Volkwein, S. 2002: Analysis of instantaneous control for Burgers equation. Nonlinear Analysis 50, 1-26.

Krstić, M. 1999: On global stabilization of Burgers equation by boundary control. Systems and Control Letters 37, 123-41.

Liu, W.-J. and Krstić, M. 2000: Backstepping boundary control of Burgers equation with actuator dynamics. Systems and Control Letter 41, 291-303.

Liu, W.-J. and Krstić, M. 2001: Adaptive control of Burgers equation with unknown viscosity. International Journal Adaptive Control and Signal Processing 15, 745-66.

McDonough, J.M. and Huang, M.T. 2004: A 'poor man's Navier-Stokes equation': derivation and numerical experiments - the 2-D case. International Journal for Numerical Methods in Fluids 44, 545-78.

Nishinari, K., Matsukidaira, J. and Takahashi, D. 2001: Two-dimensional Burgers cellular automaton. Journal of the Physical Society of Japan 70, 2267-72.

Park, H.M. and Jang, Y.D. 2002: Control of Burgers equation by means of mode reduction. International Journal of Engineering Science 38, 785-805.

Sirendaoreji, S.J. 1999: Exact solutions of the two-dimensional Burgers equation. Journal of Physics A: Mathematical and General 32, 6897-900.

Vedantham, R. 2000: Optimal control of the viscous Burgers equation using an equivalent index method. Journal of Global Optimization 18, 255-63.

Wescott, B.L. and Rizwan-uddin. 2001: An efficient formulation of the modified nodal integral method and application to the twodimensional Burgers equation. Nuclear Science and Engineering 139, 293-305.

Zhu, Z. 1996: Exact solutions for a two-dimensional KdV-Burgers-type equation. Chinese Journal of Physics 34, 1101-105. 